\title{
Artigo/Article
}

\section{Padronização do diagnóstico de Blastocystis hominis por diferentes técnicas de coloração}

\author{
Standardization of Blastocystis hominis diagnosis using different staining techniques
}

Dayane Eymael ${ }^{1}$, Graziela Maria Schuh ${ }^{2}$ e Rejane Giacomelli Tavares ${ }^{3,4}$

\section{RESUMO}

Introdução: O presente trabalho foi realizado no período de março a maio de 2008, com o objetivo de avaliar a eficácia de diferentes técnicas para diagnóstico de Blastocystis hominis em uma amostra da população atendida pelo Laboratório de Biomedicina da Universidade Feevale, Novo Hamburgo/RS. Métodos: Foram estudadas 100 amostras de fezes de crianças e adultos, que foram coletadas e submetidas às técnicas de sedimentação espontânea (HPJ), sedimentação em formol-éter (Ritchie) e de coloração por Gram e May-Grünwald-Giemsa (MGG). Resultados: A presença de Blastocystis hominis foi observada em 40 amostras, quando utilizadas técnicas de coloração (MGG e Gram), enquanto que as técnicas de sedimentação se mostraram menos eficientes ( 32 amostras positivas para a técnica de Ritchie e 20 amostras positivas para a técnica de HPJ). Conclusões: Nossos resultados demonstram que o HPJ foi menos eficiente que outros métodos, indicando a necessidade de se incluir na rotina do laboratório técnicas que permitam a identificação deste parasita.

Palavras-chaves: Blastocystis hominis. Parasitas intestinais. Diagnóstico. Técnicas de coloração.

\begin{abstract}
Introduction: The present study was carried out from March to May 2008, with the aim of evaluating the effectiveness of different techniques for diagnosing Blastocystis hominis in a sample of the population attended at the Biomedicine Laboratory of Feevale University, Novo Hamburgo, Rio Grande do Sul. Methods: On hundred feces samples from children and adults were evaluated. After collection, the samples were subjected to the techniques of spontaneous sedimentation (HPJ), sedimentation in formalin-ether (Ritchie) and staining by means of Gram and May-Grünwald-Giemsa (MGG). Results: The presence of Blastocystis hominis was observed in 40 samples, when staining techniques were used (MGG and Gram), while sedimentation techniques were less efficient ( 32 positive samples using the Ritchie technique and 20 positive samples using the HPJ technique). Conclusions: Our results demonstrate that HPJ was less efficient than the other methods, thus indicating the need to include laboratory techniques that enable parasite identification on a routine basis.
\end{abstract}

Key-words: Blastocystis hominis. Intestinal parasites. Diagnosis. Staining techniques.

1. Laboratório de Biomedicina, Setor de Parasitologia, Universidade Feevale, Novo Hamburgo, RS 2. Curso de Biomedicina, Instituto de Ciências Biológicas, Centro Universitário Franciscano, Santa Maria, RS. 3. Curso de Biomedicina, Instituto de Ciências da Saúde, Universidade Feevale, Novo Hamburgo, RS. 4. Departamento de Ciências Básicas da Saúde, Universidade Federal de Ciências da Saúde de Porto Alegre, Porto Alegre, RS.

Endereço para correspondência: Prof ${ }^{a}$. Rejane Giacomelli Tavares. Av. Grécia 1100/1805, Bairro Passo da Areia, 91350-070 Porto Alegre, RS.

Tel: 5551 3586-8800 ramal 9019

e-mail: rejanetavares@feevale.br

Recebido para publicação em 12/05/2009

Aceito em 12/04/2010

\section{INTRODUÇÃO}

Blastocystis hominis é um protozoário intestinal anaeróbico encontrado em muitas espécies animais, inclusive humanos. Esse organismo unicelular foi descrito primeiramente como um fungo por Brumpt, em 1912, foi re-classificado e incluído entre os protozoários após os estudos de Zierdt \& Tan, em $1976^{1,2}$. Recentemente, Blastocystis hominis foi classificado como um membro da família Stramenopiles. Embora muito pouco se saiba sobre o significado desse parasita na saúde pública, ele é considerado o mais comum dos protozoários reportados em amostras fecais humanas, desenvolvendo infecções em mais de 50\% dos portadores em países em desenvolvimento, e uma taxa um pouco menor nos países desenvolvidos ${ }^{3-7}$.

No Brasil, sua presença já foi relatada em diversos estudos, seja em portadores hígidos ou em imunodeprimidos ${ }^{1,7,8-11}$.

Em relação à sua classificação, estudos moleculares, utilizando reações de polimerização em cadeia (PCR), classificaram as diversas espécies de Blastocystis isoladas em aves e mamíferos em nove subtipos ( 1 a 9), de acordo com a análise das sequências de subunidades ribossomais pequenas (SSU rRNA) $)^{3,12}$.

Seu ciclo está compreendido basicamente pela contaminação fecal-oral. É um organismo polimórfico, com quatro formas principais descritas: vacuolar, granular, amebóide e cística, além das formas multivacuolar e avacuolar ${ }^{7,13,14}$. A forma vacuolar é a encontrada na maioria das vezes em fezes ${ }^{15}$. Esta se apresenta esférica, com vacúolo central, diâmetro de 6 a 15 micras e apresenta uma camada superficial de espessura variável ${ }^{6,13}$. Alguns estudos relatam a forma morfológica encontrada com a existência ou não de sintomatologia, sendo a forma vacuolar encontrada tanto em pacientes com sintomas, bem como naqueles com ausência de sintomas. Já as formas granulares, são mais relacionadas aos portadores assintomáticos, e a 
forma amebóide sempre é encontrada em pacientes sintomáticos ${ }^{13,15}$. Entretanto, essa forma é extremamente lábil e muito raramente pode ser observada nas fezes ${ }^{13}$.

Há controvérsias quanto à patogenicidade desse parasita. Alguns autores citam que em situações de alta carga parasitária de Blastocystis hominis e na ausência de outros parasitas, vírus ou bactérias, esse protozoário pode causar sintomas como diarréias, cólicas, náuseas, febres, vômitos e desconforto abdominal. Em pacientes imunodeprimidos, o quadro ainda torna-se mais intenso $0^{6,13,16}$. Outros autores afirmam que é preciso analisar muito bem os sintomas, pois são os mesmos de outras doenças, infecciosas ou não ${ }^{17}$.

Vários estudos indicam que os métodos de sedimentação espontânea ou de flutuação são ineficazes na busca de Blastocystis hominis nas fezes, pois o contato deste protozoário com água acarretaria a lise do mesmo, produzindo resultados falsonegativos ${ }^{9-11,17}$. Uma alternativa para melhorar o desempenho destas técnicas parece ser a conservação prévia das mesmas com formol ou outro tipo de conservante ${ }^{18}$.

Esta hipótese ainda é controversa, pois outro estudo demonstrou que não foi encontrada diferença nos resultados em amostras de fezes diluídas em água ou em solução fisiológica ${ }^{1}$. Estes métodos se baseiam na sedimentação das fezes, seja espontânea ou por centrifugação, onde o sedimento é avaliado através da coloração com lugol ${ }^{19}$.

Outros autores sugerem que, para obter uma identificação adequada deste parasita, sejam utilizadas técnicas de coloração, como a coloração por tricrômio, hematoxilina férrica, Giemsa, Gram ou Wright ${ }^{13}$.

O diagnóstico imunológico, através da detecção de antígenos fecais ou anticorpos ainda não se encontra disponível para aplicação clínica, muito embora já existam estudos nesse sentido. Da mesma forma, outros métodos como avaliação de fluídos obtidos por aspiração de secreções duodenais, endoscopia, sigmoidoscopia ou cultura de material fecal não são indicados, devido ao fato de serem extremamente invasivos e não apresentarem total eficácia na detecção do parasita $^{13}$.

A grande maioria dos autores encontra consenso quando afirma que a dificuldade na identificação deste protozoário está no treinamento adequado dos analistas clínicos, que possuem dificuldade em reconhecer e identificar o Blastocystis hominis ou, ainda, não atribuem a devida importância ao mesmo, não o mencionando nos resultados dos exames ${ }^{7,9,10,17}$.

Devido à frequência com que esse parasita tem sido encontrado na população, destaca-se a importância da padronização de técnicas parasitológicas que permitam o diagnóstico do Blastocystis hominis, através da sua correta identificação ${ }^{1,20}$.

\section{MÉTODOS}

De março a maio de 2008, foram analisadas 100 amostras de fezes, espontaneamente emitidas e preservadas em frasco contendo formol, encaminhadas ao Setor de Parasitologia do Laboratório de Biomedicina da Universidade Feevale.

Cada amostra fecal foi dividida em duas partes: a primeira parte foi submetida ao método de sedimentação espontânea $(\mathrm{HPJ})^{19}$ e a segunda parte foi submetida ao método de centrífugo-sedimentação em formol-éter (Ritchie) ${ }^{19}$. O sedimento resultante da técnica de Ritchie foi dividido em duas partes, sendo a primeira avaliada em microscopia com lugol, e a segunda parte utilizada para confecção de esfregaços fixados com metanol, que foram corados através das técnicas de May-Grünwald-Giemsa (MGG) e Gram, para avaliação da existência de Blastocystis hominis ${ }^{11}$. Para as técnicas de sedimentação, foram avaliadas 3 lâminas (coradas com lugol) e para as técnicas de coloração permanente, foram confeccionadas duas lâminas, sendo uma delas corada por MGG e outra por Gram, e ambas foram avaliadas microscopicamente $\mathrm{e}^{8,21,22}$.

Para validação dos resultados, em todas as técnicas foi utilizado um pool, sabidamente positivo para o parasita em questão, como forma de controle de qualidade. Da mesma forma, os reagentes usados na coloração tiveram seu controle de qualidade realizado, através da avaliação da data de validade e características físico-químicas.

Para análise estatística utilizou-se um teste não-paramétrico, o teste qui-quadrado.

\section{RESULTADOS}

Inicialmente todas as 100 amostras foram submetidas a quatro técnicas de análise: HPJ, Ritchie, coloração por MGG e coloração por Gram. Deste total, 22 foram negativas e 78 foram positivas para presença de parasitos (Tabela 1). Em relação aos protozoários, Endolimax nana foi a mais prevalente, sendo observada em 46 amostras, o que corresponde a $58,9 \%$ das amostras positivas. Blastocystis hominis foi encontrado em 40 amostras analisadas, sendo encontrado em $51,2 \%$ das amostras positivas. Outros protozoários encontrados foram Entamoeba coli (22 amostras) e Giardia lamblia (7 amostras). Dentre os helmintos, observou-se a maior prevalência de Ascaris lumbricoides, em 9 amostras (Tabela 2).

Segundo a Tabela 3, podemos observar que utilizando-se técnicas de coloração (MGG e Gram), a identificação de Blastocystis hominis foi mais efetiva do que quando comparada com os métodos de sedimentação convencionais, especialmente o HPJ. Das 40 amostras positivas para Blastocystis hominis, identificadas por coloração permanente, somente 20 foram também identificadas pela técnica de HPJ e 32 pela técnica de Ritchie. Importante ressaltar

TABELA 1 - Frequência de parasitas intestinais nas amostras analisadas $\left(\mathbf{n}^{\mathrm{o}}=\mathbf{1 0 0}\right)$.

\begin{tabular}{lrr}
\hline Presença de Parasitas & Número & Percentagem \\
\hline Positivos & 78 & 78,0 \\
Negativos & 22 & 22,0 \\
\hline Total & $\mathbf{1 0 0}$ & $\mathbf{1 0 0 , 0}$ \\
\hline
\end{tabular}

TABELA 2 - Distribuição dos parasitas intestinais nas amostras positivas* $\left(\mathrm{n}^{\mathrm{o}}=78\right)$.

\begin{tabular}{lcc}
\hline & \multicolumn{2}{c}{ Amostras positivas } \\
\cline { 2 - 3 } Parasitas & $\mathrm{n}^{\circ}$ & $\%$ \\
\hline Protozoários & 46 & 58,9 \\
$\quad$ Endolimax nana & 40 & 51,2 \\
Blastocystis hominis & 22 & 28,2 \\
Entamoeba coli & 7 & 8,9 \\
Giardia lamblia & & \\
Helmintos & 9 & 11,5 \\
Ascaris lumbricoides & 4 & 5,1 \\
Trichuris trichuira & 1 & 1,3 \\
Strongyloides stercoralis & & \\
\hline
\end{tabular}

*dados incluem amostras positivas monoparasitadas e poliparasitadas. 
TABELA 3 - Distribuição dos parasitas intestinais nas amostras positivas, de acordo com o método utilizado: sedimentação espontânea em água (HPJ), sedimentação em formalina-éter (Ritchie), Coloração por MGG e Coloração por Gram $\left(\mathbf{n}^{\mathrm{o}}=78\right)$.

\begin{tabular}{|c|c|c|c|c|c|c|c|c|c|}
\hline \multirow[b]{3}{*}{ Parasitos } & \multicolumn{9}{|c|}{ Método Utilizado } \\
\hline & \multicolumn{2}{|c|}{ Total } & HPJ & \multicolumn{2}{|c|}{ Ritchie } & \multicolumn{2}{|c|}{ MGG } & \multicolumn{2}{|c|}{ Gram } \\
\hline & $\mathrm{n}^{\circ}$ & $\%$ & $\mathrm{n}^{\mathrm{o}}$ & $\mathrm{n}^{\mathrm{o}}$ & $\%$ & $\mathrm{n}^{\mathrm{o}}$ & $\%$ & $\mathrm{n}^{\mathrm{o}}$ & $\%$ \\
\hline \multicolumn{10}{|l|}{ Protozoários } \\
\hline Endolimax nana & 46 & 100,0 & 4189,0 & 46 & 100,0 & 27 & 58,0 & 31 & 67,0 \\
\hline Blastocystis hominis & 40 & 100,0 & 2050,0 & $32^{*}$ & 80,0 & $40^{*}$ & 100,0 & $40^{*}$ & 100,0 \\
\hline Entamoeba coli & 22 & 100,0 & 22100,0 & 20 & 91,0 & 18 & 81,0 & 18 & 81,0 \\
\hline Giardia lamblia & 7 & 100,0 & 457,0 & 7 & 100,0 & 5 & 71,0 & 5 & 71,0 \\
\hline \multicolumn{10}{|l|}{ Helmintos } \\
\hline Ascaris lumbricoides & & 100,0 & 9100,0 & 9 & 100,0 & 6 & 67,0 & 5 & 55,0 \\
\hline Trichuris trichuira & & 100,0 & 4100,0 & 3 & 75,0 & 2 & 50,0 & 2 & 50,0 \\
\hline Strongyloides stercoralis & is 1 & 100,0 & 1100,0 & 0 & 0,0 & 0 & 0,0 & 0 & 0,0 \\
\hline
\end{tabular}

que todas as 20 amostras positivas na técnica de HPJ, também, foram positivas na técnica de Ritchie. Entretanto, se observou diferença estatística significativa entre as duas técnicas de sedimentação (HPJ e Ritchie), bem como quando comparadas as técnicas de coloração (MGG e Gram) com a técnica de HPJ. Quando comparadas as técnicas de coloração utilizadas (Gram e MGG), percebe-se que ambas apresentam eficiência semelhante, conforme já descrito na Tabela 3.

Na Figura 1, podemos comparar as imagens obtidas com as diferentes colorações utilizadas.

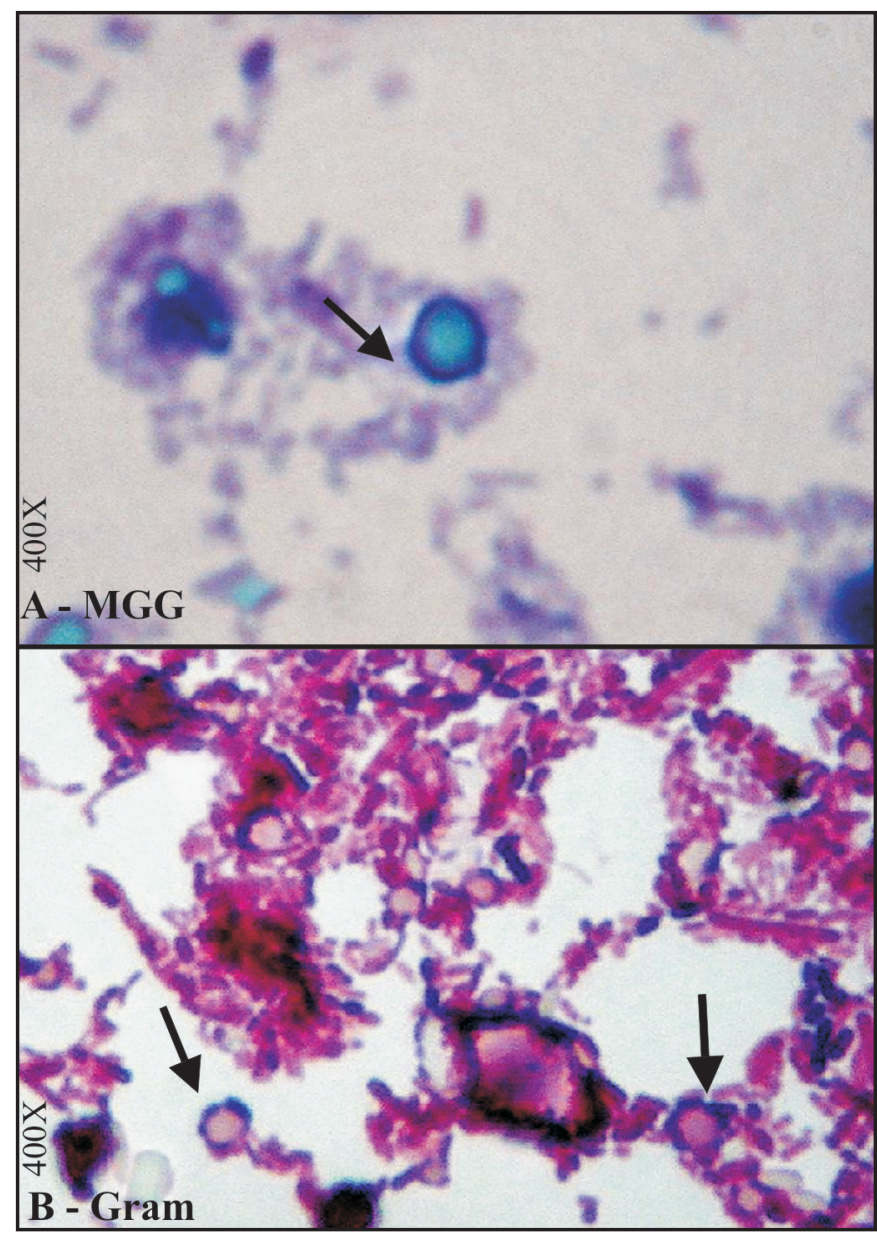

FIGURA 1 - Visualização do Blastocystis hominis nas diferentes técnicas de coloração utilizadas. A: MGG. B: Gram.

\section{DISCUSSÃO}

Vários estudos sobre a importância do Blastocystis hominis em patologias humanas têm sido realizados ${ }^{1,3,6,8,9,12,14,23}$. Entretanto, seu papel exato ainda não se encontra perfeitamente elucidado. Não está categoricamente provado que esse protozoário seja comensal. Por outro lado, há relatos que consideram a possibilidade dele causar manifestações clínicas, sobretudo quando excluídos outros agentes tidos como patogênicos. Outrossim, julgam que a quantidade encontrada influi, em especial, nos indivíduos imunocomprometidos ${ }^{9,10}$. Alguns autores sugerem que esse deva ser considerado patogênico somente em pessoas sintomáticas, especialmente crianças, ou quando o número de organismos no exame microscópico das fezes excederem cinco organismos por campo ${ }^{4}$.

Grande parte dessa confusão se deve à dificuldade de execução do diagnóstico adequado. Segundo Amato Neto e cols (2004), sua visualização só poderia ser realizada com o uso do exame direto ou de colorações permanentes, como hematoxilina férrica, tricrômio ou da tionina ${ }^{10}$. Já segundo Velásquez e cols (2005), a preservação das amostras com formol a $10 \%$ permite o diagnóstico utilizando a técnica de sedimentação espontânea em água, apesar da fragilidade das formas vacuolares ${ }^{7}$. Resultados semelhantes foram relatados por Martins e cols (2007), onde foi possível a visualização do protozoário em preparações utilizando água ${ }^{1}$.

Embora as técnicas rotineiras sejam de mais fácil execução, muitas vezes a falta de treinamento dos analistas pode gerar dificuldades no diagnóstico. Para facilitar a observação, em nosso laboratório avaliamos quatro métodos de diagnóstico: dois rotineiramente utilizados (HPJ e Ritchie) e dois métodos de coloração permanente (MGG e Gram).

Em relação aos métodos de sedimentação, nosso estudo demonstrou que a técnica de centrífugo-sedimentação foi mais efetiva no isolamento de protozoários, especialmente de Endolimax nana e Blastocystis hominis do que a técnica de HPJ (Tabela 3 ). Esse achado pode ser resultante da força centrífuga aplicada, que possivelmente força a deposição da forma vacuolar do protozoário. Além disso, a utilização de formalina-éter promove a obtenção de um sedimento um pouco mais purificado do que a sedimentação espontânea, facilitando a análise ${ }^{21,24}$.

Semelhante ao relatado por Amato Neto e cols (2004), a identificação do Blastocystis hominis se tornou extremamente facilitada com a utilização de técnicas de coloração permanente, muito embora não se tenha utilizado a coloração por hematoxilina férrica (Figura 1 $)^{9,10}$. Como vantagens da utilização de qualquer uma das técnicas de coloração permanente (MGG e Gram), podemos citar a sua utilização rotineira nos laboratórios clínicos, seja no setor de Hematologia ou no setor de Bacteriologia, o que dispensa o gasto econômico do laboratório com a montagem de uma técnica de coloração específica ${ }^{25}$. Observamos também que utilizando o método de coloração de MGG há uma maior facilidade, já que, durante a coloração de Gram, temos a etapa da descoloração que é crítica, pois a exposição prolongada ao solvente pode provocar a remoção do cristal violeta do interior dos núcleos periféricos do Blastocystis hominis, ou ainda, se não houver a descoloração necessária não iremos observar o vacúolo central, prejudicando assim a visualização do parasita em questão ${ }^{22,26}$. 
Cabe ressaltar que o sucesso na identificação de Blastocystis hominis, utilizando-se técnicas de coloração, foi favorecido pela utilização de sedimentos obtidos pela técnica de Ritchie, que certamente promoveu uma maior concentração dos espécimes parasitários, além de eliminar gotículas de gordura, que poderiam ser confundidas com o parasita, em função de forma e tamanho ${ }^{21,24}$.

Embora o método de coloração seja um processo mais demorado e trabalhoso que os métodos convencionais utilizados, muitas publicações relatam que este é o método mais sensível para a visualização de Blastocystis hominis, o que evidencia a necessidade de se incluir a coloração na rotina do laboratório, evitando assim a ocorrência de resultados falso-negativos $9,13,17,27,28$.

A blastocistose ainda permanece cercada de controvérsias e incertezas, sobretudo referente à patogenicidade. Enquanto alguns autores sustentam a idéia de que a infecção por Blastocystis hominis é autolimitada e não deve ser tratada, outros afirmam que sua frequência e patogenicidade são realmente significativas. Assim, é importante e desejável que conhecimentos adequados sejam satisfatoriamente divulgados, para evitar procedimentos impróprios e auxiliar no sentido de tornar ampla a difusão de corretas particularidades vinculadas a tal microorganismo.

\section{CONFLITO DE INTERESSE}

Os autores declaram não haver nenhum tipo de conflito de interesse no desenvolvimento do estudo.

\section{REFERÊNCIAS}

1. Martins LPA, Serapião AATB, Valenciano RF, Pires JEC, Castanho REP. Freqüência de Blastocystis hominis e outras enteroparasitoses em amostras fecais analisadas no laboratório de patologia da Faculdade de Medicina de Marília - SP. Rev Pat Trop 2007; 36:47-53.

2. Silberman JD, Sogin ML, Leipe DD, Clark CG. Human parasite finds taxonomic home. Nature 1996; 380:398.

3. Menounos PG, Spanakos G, Tegos N, Vassalos CM, Papadopoulou C, Vakalis NC. Direct detection of Blastocystis sp. in human faecal sample and subtype assignment using single strand conformational polymorphism and sequencing. Mol Cel Probes 2007; 22:24-29.

4. Nimri LS. Evidence of an epidemic of Blastocystis hominis infections in preschool children in Northern Jordan. J Clin Microbiol 1993; 31:2706-2708.

5. Noël C, Dufernez F, Gerbod D, Edgcomb VP, Viscogliosi PD, Ho LC, Singh M, et al. Molecular phylogenies of Blastocystis isolates from different hosts: Implications for genetic diversity, Identification of species, and zoonosis. J Clin Microbiol 2005; 43:348-355.

6. Silva AA. Incidência de Blastocystis hominis na população da cidade do Rio de Janeiro, RJ. Newslab 2006; 76:86-96.

7. Velásquez V, Caldera R, Wong W, Cermeño G, Fuentes M, Blanco Y, et al. Elevada prevalência de blastocistose em pacientes do Centro de Saúde de Soledad, Estado Anzoátegui, Venezuela. Rev Soc Bras Med Trop 2005; 38:356-357.

8. Aguiar JIA, Gonçalves AQ, Sodré FC, Pereira SR, Bóia MN, Lemos ERS, et al. Intestinal protozoa and helminths among Terena Indians in the state of Mato Grosso do Sul: high prevalence of Blastocystis hominis. Rev Soc Bras Med Trop 2007; 40:631-634

9. Alarcón RSR, Amato Neto V, Gakiya E, Bezerra RC. Observações sobre Blastocystis hominis e Cyclospora cayetanensis em exames parasitológicos efetuados rotineiramente. Rev Soc Med Trop 2007; 40:253-255.

10. Amato Neto V, Alarcón RSR, Gakiya E, Ferreira SC, Bezerra RC, Santos AG. Elevada porcentagem de blastocistose em escolares de São Paulo - SP. Rev Soc Bras Med Trop 2004; 37:354-356.
11. Nascimento SA, Moitinho MLR. Blastocystis hominis and other intestinal parasites in a community of Pitanga city, Paraná State, Brazil. Rev Soc Bras Med Trop 2005; 47:213-217.

12. Stensvold AR, Suresh GK, Tan KSW, Thompson RCA, Traub RJ, Viscogliosi E, et al. Terminology for Blastocystis subtipes - a consensus. Trends Parasitolol 2007; 23:93-96.

13. Stenzel DJ, Boreham PFL. Blastocystis hominis revisited. Clin Microbiol Ver 1996; 9: 563-584.

14. Yaicharoen R, Ngrenngarmlert W, Wongjindanon N, Sripochang S, Kiatfuengfoo $\mathrm{R}$. Infection of Blastocystis hominis in primary schoolchildren from Nakhom Pathom province, Thailand. Trop Biomed 2006; 23:117-122.

15. Tan TC, Suresh KG. Predominance of amoeboid forms of Blastocystis hominis in isolate from symptomatic patients. Parasitol Res 2006; 98:189-193.

16. Teixeira ATLS, Garlipp CR, Bottini PV, Souza R. Blastocystis hominis: Prevalência e patogenicidade. Rev Bras Patol Clin 1989; 25:7-9.

17. Amato Neto V, Alarcón RSR, Gakiya E, Bezerra RC, Ferreira CS, Braz LMA. Blastocistose: controvérsias e indefinições. Rev Soc Bras Med Trop $2003 ; 36: 515-517$.

18. Atlas of Medical Parasitology. Disponível em <http://www.cdfound.to.it/ HTML/atlas.htm>. Acesso em: 12 nov. 2009.

19. De Carli GA. Diagnóstico laboratorial das parasitoses humanas. Rio de Janeiro: Medsi; 1994.

20. Kobayashi J, Hasegawa H, Forli AA, Nishimura NF, Yamanaka A, Shimabukuro $\mathrm{T}$, et al. Prevalence of intestinal parasitic infection in five farms in Holambra, São Paulo, Brazil. Rev Inst Med Trop Sao Paulo 2005; 37:13-18.

21. Barassa B, Bueno VS. Estudo comparativo entre os métodos de centrífugoflutuação e de centrífugo-sedimentação no diagnóstico coproparasitológico. Lecta-USF 2000; 18:65-73.

22. Freitas VR, Picoli SU. A coloração de Gram e as variações na sua execução. Newslab 2007; 82:124-128.

23. Özyurt M, Kurt Ö, Mølbak K, Nielsen HV, Haznedaroglu T, Stensvold CR. Molecular epidemiology of Blastocystis infections in Turkey. Parasitol Int 2008; 57: 300-306

24. Waldman E, Tzipori S, Forsyth JRL. Separation of Cryptosporidium species oocysts from feces by using a percoll discontinuous density gradient. J Clin Microbiol 1986; 23:199-200.

25. Oliveira RAG, Poli Neto A. Anemias e leucemias. Conceito básico e diagnóstico por técnicas laboratoriais. São Paulo: Roca; 2004.

26. Núñez FA, Ginorio DE, Cordoví RA, Finlay CM. Intervención educativa para mejorar la calidad del diagnóstico coproparasitológico em la red de salud de ciudad habana, Cuba. Cad Saude Publica 1998; 14:139-144.

27. Qadri SMH, Al-Okaili GA, Al-Dayel F. Clinical Significance of Blastocystis hominis J Clin Microbiol 1989; 27:2407-2409.

28. Babb RR, Wagener S. Blastocystis hominis - A potential intestinal pathogen. West J Med 1989; 151:518-519. 